\title{
Preparation of intercalated polyaniline/clay nanocomposite and its exfoliation exhibiting dendritic structure
}

\author{
N SRIVASTAVA ${ }^{1,2}$, Y SINGH ${ }^{1, *}$ and R A SINGH ${ }^{2}$ \\ ${ }^{1}$ Department of Chemistry, Udai Pratap College, Varanasi 221 002, India \\ ${ }^{2}$ Department of Chemistry, Faculty of Science, Banaras Hindu University, Varanasi 221 010, India
}

MS received 14 March 2010; revised 18 April 2010

\begin{abstract}
Intercalated composite of polyaniline and clay has been reported. The composite was prepared by in situ polymerization of aniline within the layers of 'illite' clay. The composite was characterized for its structural, spectral, and microscopic properties. At higher level of loading the layered structure of composite breaks forming exfoliated composite, revealing well-defined nanosized dendritic morphology of polyaniline.
\end{abstract}

Keywords. Polyaniline; illite; nanocomposite; exfoliated composite.

\section{Introduction}

Inorganic-organic nanostructured materials have become a field of intensive interest for scientists and industrialists due to their multifaceted properties (Gomez-Romero 2001). Recently, a new class of advanced materials based on conducting polymer-clay nanocomposites has added a novel dimension to this field (Skotheim et al 1998; Chandrasekhar 1999; Gangopadhyay and De 2000). These materials have given manifold high-tech applications on electrorheological fluids, anti-corrosion materials, molecular wires, sensor devices, smart windows, electochemical devices etc (Pinnavaia and Beall 2001).

Clays are natural substances having interesting properties due to their geometries, surface area and electrostatic charge. Incorporation of guest electroactive polymers such as polyaniline (PANI), polypyrrole (PPy) etc. into host clay particles has attracted great attention because of their better processibilty with colloidal stability, mechanical strength and novel electrical, catalytic properties (Alexandre and Dubois 2000). Intercalation and delamination methods have been used extensively to explain the nanomorphologies of composites (Kanatzidis et al 1987; Carrado and $\mathrm{Xu}$ 1998).

Intercalation of electronically conducting polymer in clays is widely studied by various scientists. Kanatzidis et al (1987) have reported first time a procedure termed 'in situ intercalative polymerization' for preparing $\mathrm{PPy}-\mathrm{FeOCl}$ nanocomposites. The interlayer adsorption of aniline into $\mathrm{Cu}^{2+}$ and $\mathrm{Fe}^{2+}$ montmorillonites from aqueous solutions was studied by Cloos and coworkers

*Author for correspondence (prrasingh@gmail.com)
(Moreale et al 1985; Cloos et al 1979). Mehrotra and Giannelis have reported the intercalative polymerization of aniline using a $\mathrm{Cu}^{2+}$ exchanged synthetic hectorite but these nanocomposites are insulating form of PANI. The conducting form was obtained by exposing these composites to $\mathrm{HCl}$ vapors (Mahrota and Giannelis 1991). In another general method PANI was formed in the interlayer space of smectites by an ion-exchange process (Chang et al 1992). Synthesis of same compounds was reported by electrochemical procedure also (Ioune and Yoneyama 1987). The conductivity of PANI-clay composite prepared from anilinium-montmorillonite was found lower than the nanocomposites reported by Mahrota and Giannelis which was assumed due to high anisotropic conductivity in these materials. Carado and $\mathrm{Xu}$ have proposed another approach to synthesize PANI-hectorite nanocomposites by in situ hydrothermal crystallization (Carrado and Xu 1998). Recently, a solvent-free synthesis of polyaniline-clay nanocomposite is reported by mechanochemical intercalation method (Yoshimoto et al 2004). The electrorheological property of polyanilinemontmorillonite clay has been investigated extensively (Choi et al 2001; Lua and Zhao 2002). Apart from PANIclay nanocomposites, many other systems have also been synthesized based on intercalation method (Mortland and Pinnavaia 1971). Besides intercalated composites, delaminated clay layers resulting into exfoliated structure have also attracted interest due to enhanced interaction of polymer-clay (Narayanan et al 2010). Exfoliated nanocomposites are more interesting because entire surface of clay layers could be used by polymer. Recently, some works have been reported regarding the enhancement of conductivity (Gök et al 2007; Bekri-Abbes and Srasra 2010) and anti-corrosive (Olad and Rashidzadeh 2008; 
Olad and Naseri 2009) properties in pani/clay composite. They showed that conductivity of pani/montmorillonite is higher than pure polyaniline due to polymerization of aniline in the clay interlayer with an extended chain conformation which effect the decreasing of $\pi$ defects and the polymer bridges.

Our current work focuses on the enhancement of structural properties of PANI. We selected clay of river Ganga as host material due to its small particle size and easy availability. Aniline monomer acted as guest material. The composite was synthesized by in situ chemical polymerization method.

\section{Experimental}

Aniline (qualigen AR) was distilled prior to use. The clay was collected at a depth of two feet on the bank of river Ganga and a distance of five feet from the running water. The clay and PANI composites were made in 5, 10, 20, $40,60,80$ PANI wt $\%$. The monomer solution was made by adding $0.25,0.50,1.00,2.00,3.00$ and $4.00 \mathrm{ml}$ (separately) of pristine aniline (specific gravity 1.02 ) to $100 \mathrm{ml}$ of $0.5 \mathrm{M} \mathrm{HCl}$. Required amount of washed and dried clay $(4 \cdot 75,4 \cdot 50,4 \cdot 0,3 \cdot 0,2.0$ and $1.0 \mathrm{~g}$ respectively) was added to the aniline hydrochloride solution and stirred continuously for $3-4 \mathrm{~h}$ in ice-bath. Oxidizing agent $\left(\mathrm{NH}_{4}\right)_{2} \mathrm{~S}_{2} \mathrm{O}_{8} \quad(0.2 \mathrm{M}) \quad$ (qualigen) was added slowly to start the polymerization. After completion of polymerization the colour of solution turned green. The solution was stirred on magnetic stirrer for 3-4 $\mathrm{h}$. The mixture was left overnight at about $4-6^{\circ} \mathrm{C}$. Next day, the suspension was poured off and the compact sediment of composite was redispersed in $0.2 \mathrm{M} \mathrm{HCl}$. After the sedimentation of clay, the supernatant liquid was again poured off. The product was washed with $0 \cdot 2 \mathrm{M} \mathrm{HCl}$ followed by distilled water repeatedly. The filtered powder was dried in oven at $50^{\circ} \mathrm{C}$ and characterized.

The energy dispersive X-ray analysis (EDAX) for elemental analysis was done using X-ray microanalyzer (Oxford Scientific). XRD were recorded on Bruker X-ray diffractometer D8 using $\mathrm{CuK}_{\alpha}$ radiation. TEM photos were taken using JEOL 2010 HRTEM at $80 \mathrm{kV}$ acceleration voltage; the samples for the TEM experiments were prepared by suspending dried samples in absolute ethanol. A drop of the sample suspension was allowed to dry on a copper grid (400 mesh, Electron Microscopy Sciences) coated with a carbon film. The a.c. electrical measurements were done on LCZ meter (Keithley Model3330 ) at various frequencies at room temperature between two platinum contacts. The samples were compressed in form of pellet at 8 ton load. The specific conductance (conductivity) was calculated by normalizing the conductance with area $\left(1.32 \mathrm{~cm}^{2}\right)$ and thickness $(4-5 \mathrm{~mm})$ of the pellet.

\section{Results and discussion}

\subsection{Mineralogy}

The energy dispersive X-ray analysis (EDAX) produced strong silicon $(1.7398 \mathrm{KeV}, \mathrm{K} \alpha)$, oxygen $(0.5249 \mathrm{KeV}$, $\mathrm{K} \alpha)$, and aluminum $(1.4866 \mathrm{KeV}, \mathrm{K} \alpha)$ peaks, with some traces of potassium $(3.3129 \mathrm{KeV}, \mathrm{K} \alpha)$, iron $(0.7048 \mathrm{KeV}$, $\mathrm{L} \alpha \mathrm{KeV}, \mathrm{K} \alpha)$, titanium $(0.4522 \mathrm{KeV}, \mathrm{L} \alpha \mathrm{KeV}, \mathrm{K} \alpha)$ and carbon $(0.2774 \mathrm{KeV}, \mathrm{K} \alpha$ (figure 1$)$. The chemical structure of illite form of clay is $\left(\mathrm{K}, \mathrm{H}_{3} \mathrm{O}\right)\left[\mathrm{Al}_{2-x}(\mathrm{Mg} . \mathrm{Fe})_{x}\right]$ $\left(\mathrm{Si}_{4-x} \mathrm{Al}_{x}\right) \mathrm{O}_{10}(\mathrm{OH})_{2}$ with majority constituent $\mathrm{SiO}_{2}$ (54.01\%) and $\mathrm{Al}_{2} \mathrm{O}_{3}$ (17.02\%) (Weaver 1973). The comparison of this information with our data demonstrates that the clay we used was in illite form. This was further confirmed by XRD study of the clay.

\subsection{XRD studies}

XRD spectra were recorded to determine the degree of swelling in clays and to monitor the formation and structure of these nanocomposites. Figure 2 shows wide as well as small angle XRD spectra of the composites. The wide angle spectra of clay show an intense peak at $2 \theta=26.72^{\circ}$ (003 plane) (figure 2c), which is characteristic of 'illite' form of clay (Usuki et al 1993). As far as polyaniline is concerned, it is a semicrystalline material, which shows broad and less intense band in XRD spectra. The characteristic band is found at $2 \theta=25.4^{\circ}$ (figure 2a) (Messersmith et al 1994). The peak is close to the peaks of illite and is not resolvable in the composite spectra due to superimposition (figure 2b). While the wide angle XRD is used to characterize the material, the low angle XRD is used to study the exfoliation mechanism of clay. The low angle spectra of pure clay (spectra ' $a$ ' in the inset of figure 2) show d001 peak at $2 \theta=9 \cdot 28^{\circ}$ (corresponding $d=9.52 \AA$ ). On intercalation (spectra ' $\mathrm{b}$ ') the d001 $2 \theta$ value moves to $7.72^{\circ}$, showing an increase in the

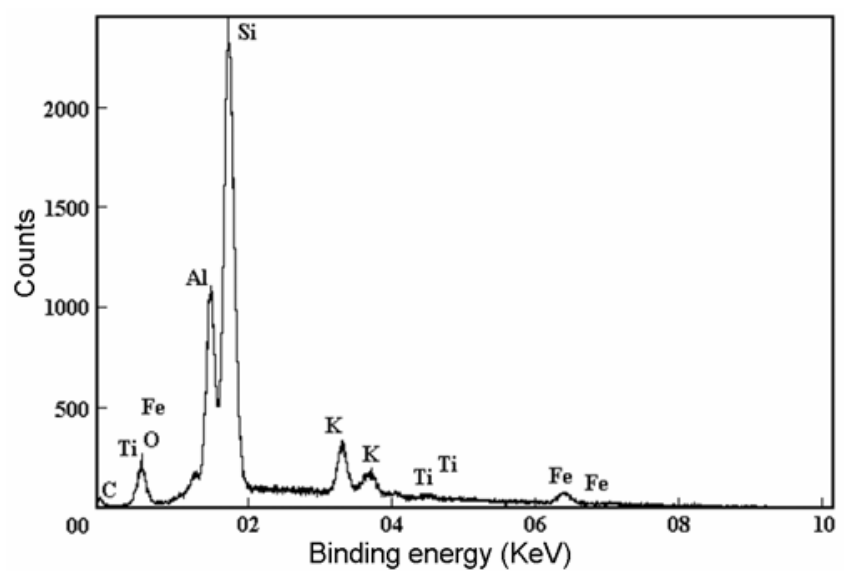

Figure 1. Energy dispersive X-ray spectra of illite 


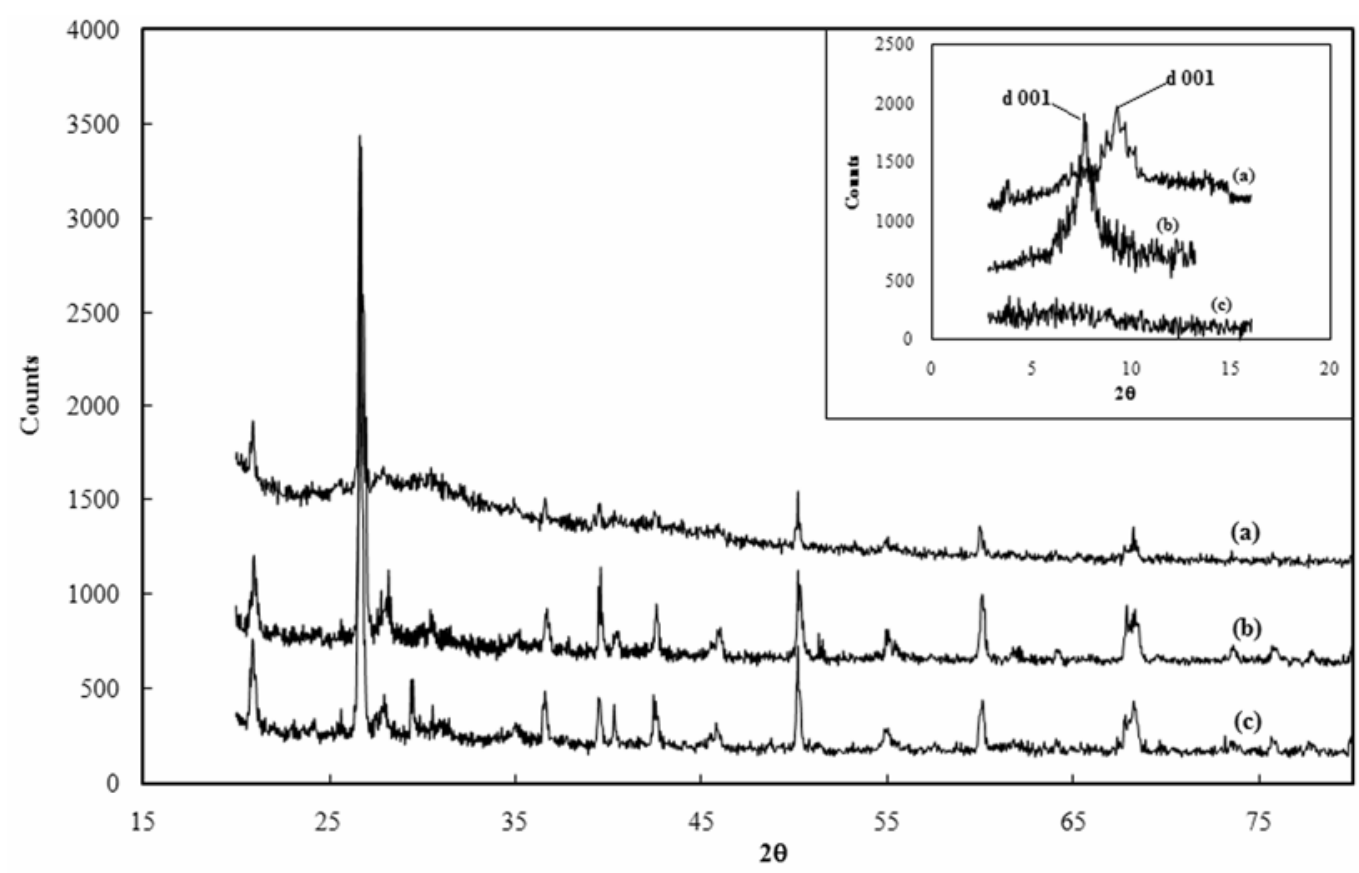

Figure 2. XRD spectra of (a) pure polyaniline; (b) polyaniline-illite (10 wt\% PANI) composite; (c) pure illite. (Inset: low angle XRD spectra of (a) pure illite; (b) polyaniline-illite (6 wt\% PANI) composite; (c) exfoliated composite 50 wt $\%$ PANI.
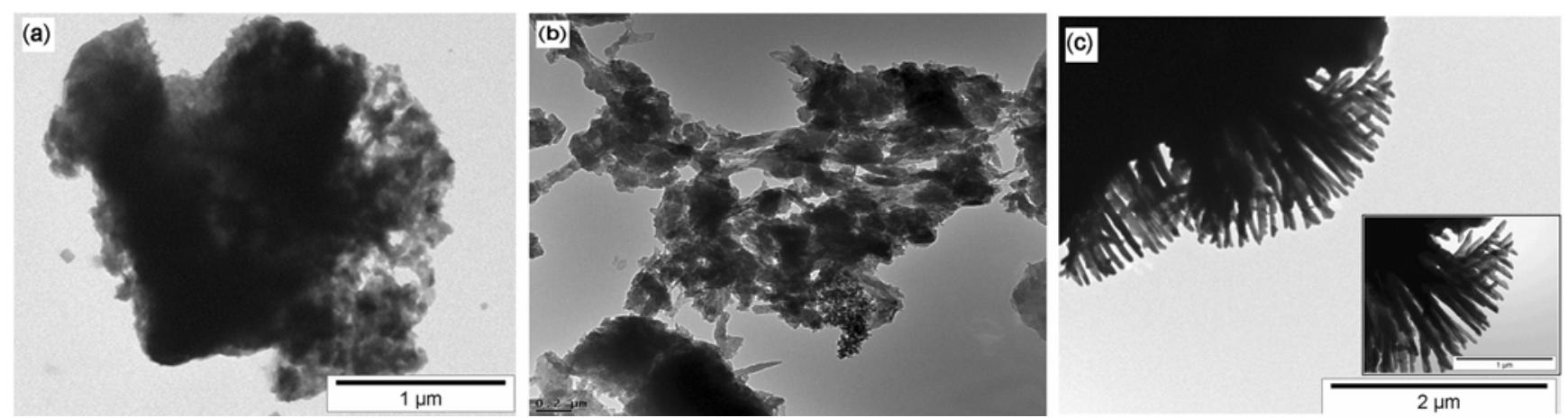

Figure 3. TEM Image of (a) intercalated composite (10 wt \% PANI); (b) partially exfoliated composite (40 wt $\%$ PANI); (c) exfoliated composite (50 wt\% PANI).

interlayer spacing up to $1 \cdot 14 \mathrm{~nm}$ in $6 \mathrm{wt} \%$ loading level. Whereas at higher loading level (spectra ' $c$ ') the gallery structure of the clay breaks due to pressure exerted by the intercalated polymer, leaving behind exfoliated composite. This leads in to disappearance of the peaks in the low angle XRD spectra.

\subsection{TEM}

TEM images are recorded for direct inspection of these composites. The TEM image of intercalated composite is given in figure $3 \mathrm{a}$, which does not show any noteworthy structure of polyaniline. As the amount of monomer (and hence polymer) increased, the composite becomes exfoli- ated (Chen 2004; Choi et al 2001). The TEM image at $40 \mathrm{wt} \%$ PANI (figure 3b) shows partially exfoliated nanocomposite. Delamination of clay galleries and beeping out of polyaniline from galleries is clearly seen at this stage. At $50 \mathrm{wt} \%$ PANI the rupture of clay galleries exposes the trapped polyaniline dendrites (figure $3 \mathrm{c}$ ). The TEM images of these exfoliated composite show well defined dendritic structure of diameter $\approx 50 \mathrm{~nm}$ and length $\approx 700 \mathrm{~nm}$ (figure $4 \mathrm{~b}$ ).

\subsection{Electrical characterization}

The mechanism of composite construction is further verified by in-phase equivalent series resistance (ESR) meas- 


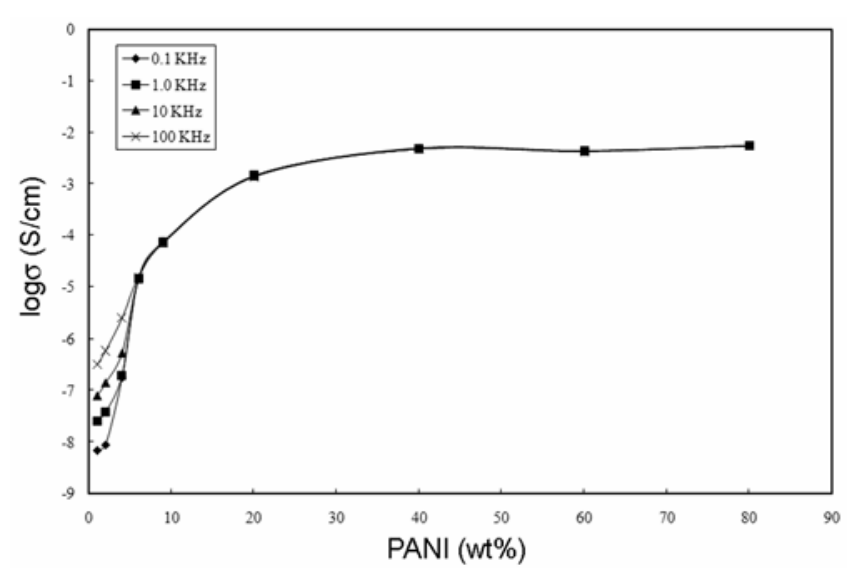

Figure 4. Variation of conductance of the composite as a function of amount of PANI loading at various frequencies $(\mathrm{Hz})$ showing percolation behaviour.

urement. Figure 4 shows a plot between log of reciprocal of ESR vs. the loading level. This plot demonstrates typical percolation behaviour, with a percolation threshold around $9 \mathrm{wt} \%$. The conductance of illite as measured by us at $100 \mathrm{~Hz}$ frequency is $3.94 \times 10^{-9} \mathrm{~S} / \mathrm{cm}$, whereas the literature value is in the range of $10^{-7}-10^{-8} \mathrm{~S} / \mathrm{cm}$ (Chen 2004). The electrical property of clay is mainly related to their ionic conductivity. The presence of hydrated cations in the interlayer space ensures conductivity in the interlayer region. Further, the conductance of the composite starts increasing rapidly as the polyaniline grows in the interlayer region. The conductance near the percolation threshold is around $10^{-3} \mathrm{~S} / \mathrm{cm}$. The percolation behaviour is observed due to formation of interpenetrating reticulate structure of the conducting polymer. At the percolation threshold the conductance of the material gets enhanced by many orders due to interconnection of conducting phase in the insulating matrix. High aspect ratio conductive fillers, like polyaniline dendrimers in current investigation, exhibit relatively low percolation threshold due to preferential alignment within the polymer matrix, which leads to anisotropic electrical properties favouring electron transport predominantly in the in-plane direction. A plateau in the conductance vs. composition plot has been observed after the percolation threshold. This behaviour is due to formation of enough channels and the number of charge carriers becoming limiting factor.

Further evidence of the netting of the polyaniline is its frequency dependence of the conductance. The conductance measured at $100,1 \mathrm{~K}, 10 \mathrm{~K}$ and $100 \mathrm{~K} \mathrm{~Hz}$ is plotted in figure 4. A little frequency dependence at lower loading level, i.e. 2-6 wt\%, may be due to interlayer capacitance and presence of hydrated cations. Formation of interconnecting polyaniline dendrites makes ohmic contacts, with disappearance of capacitive components and hence the frequency independence of conductance, at higher loading levels. Hence after percolation threshold we see superimposition of all the four plots in figure 4 .

\section{Conclusions}

The results of current investigation demonstrated formation of intercalated composite of polyaniline in clay interlayer. The intercalation and exfoliation phenomenon of clay has been studied by various characterization techniques. The layered structure of the clay completely breaks after $50 \mathrm{wt} \%$ loading of PANI, showing unique dendritic growth of polyaniline.

\section{References}

Alexandre M and Dubois P 2000 Mater. Sci Eng. R28 1

Bekri-Abbes I and Srasra E 2010 Reactive \& Funct. Poly. 7011

Carrado K A and Xu L 1998 Chem. Mater 101440

Chandrasekhar P 1999 Conducting polymers, fundamentals and applications: a practical approach (Kluwer Academic Publishers)

Chang T C et al 1992 J. Chin. Chem. Soc. 39209

Chen B 2004 Br. Ceram. T 103241

Choi H J et al 2001 Appl. Phys. Lett. 783806

Choi H J et al 2001 Synth. Met. 1211325

Cloos P et al 1979 Clay Miner. 14307

Gangopadhyay R and De A 2000 Chem. Mater. 12608

Gök A et al 2007 European Poly. J. 432471

Gomez-Romero P 2001 Adv. Mater. 13163

Ioune H and Yoneyama H 1987 J. Electroanal. Chem. 233291

Kanatzidis M G et al 1987 J. Am. Chem. Soc. 1093797

Lua J and Zhao X 2002 J. Mater. Chem. 122603

Mahrota V and Giannelis P E 1991 Solid State Commun. 77155

Messersmith P B and Giannelis E P 1994 Chem. Mater. 6 1719

Moreale A et al 1985 Clay Miner. 2029

Mortland M M and Pinnavaia T J 1971 Nature 22975

Narayanan B N et al 2010 Mater. Sci. Eng. B (doi:10.1016/ j.mseb.2009.12.027)

Olad A and Naseri B 2009 Prog. Org. Coat (doi:10.1016/ j.progcoat.2009.12.003)

Olad A and Rashidzadeh A 2008 Prog. Org. Coat. 62293

Pinnavaia T J and Beall G W 2001 Polymer-clay nanocomposites (New York: Wiley)

Skotheim T A et al 1998 Handbook of conducting polymers (New York: Marcel Dekker Inc.)

Usuki A et al 1993 J. Mater. Res. 81174

Weaver C E 1973 The chemistry of clay minerals (New York: Elsevier Scientific Publishing Company)

Yoshimoto S et al 2004 Chem. Commun. 1924 